\title{
Whole genome sequencing of a natural recombinant Toxoplasma gondii strain reveals chromosome sorting and local allelic variants Irene Lindström Bontell ${ }^{*}$, Neil Hall ${ }^{\dagger}$, Kevin E Ashelford ${ }^{\dagger}$, JP Dubey ${ }^{\ddagger}$, Jon P Boyle§, Johan Lindh" and Judith E Smith*
}

\begin{abstract}
Addresses: *Institute of Integrative and Comparative Biology, Clarendon Way, University of Leeds, Leeds, LS2 9JT, UK. 'School of Biological Sciences, University of Liverpool, Crown Street, Liverpool, L69 7ZB, UK. *United States Department of Agriculture, Agricultural Research Service, Animal and Natural Resources Institute, Animal Parasitic Diseases Laboratory, Baltimore Avenue, Beltsville, MD 20705, USA. §Department of Biological Sciences, University of Pittsburgh, Fifth Avenue, Pittsburgh, PA 1526o, USA. "Department of Parasitology, Mycology and Environmental Microbiology, Swedish Institute for Infectious Disease Control (SMI), Nobels väg, 17182 Solna, Sweden. ${ }^{¥}$ Current address: Division of Clinical Microbiology, Department of Medicine, Karolinska Institutet, Alfred Nobels Allé, 14186 Stockholm, Sweden.
\end{abstract}

Correspondence: Judith E Smith. Email: j.e.smith@leeds.ac.uk

Published: 20 May 2009

Genome Biology 2009, 10:R53 (doi:10.1 186/gb-2009-10-5-r53)

The electronic version of this article is the complete one and can be found online at http://genomebiology.com/2009/10/5/R53
Received: 27 February 2009

Revised: I May 2009

Accepted: 20 May 2009

(C) 2009 Lindström Bontell et al.; licensee BioMed Central Ltd.

This is an open access article distributed under the terms of the Creative Commons Attribution License (http://creativecommons.org/licenses/by/2.0), which permits unrestricted use, distribution, and reproduction in any medium, provided the original work is properly cited.

\begin{abstract}
Background: Toxoplasma gondii is a zoonotic parasite of global importance. In common with many protozoan parasites it has the capacity for sexual recombination, but current evidence suggests this is rarely employed. The global population structure is dominated by a small number of clonal genotypes, which exhibit biallelic variation and limited intralineage divergence. Little is known of the genotypes present in Africa despite the importance of AIDS-associated toxoplasmosis.

Results: We here present extensive sequence analysis of eight isolates from Uganda, including the whole genome sequencing of a type II/III recombinant isolate, TgCkUg2. 454 sequencing gave $84 \%$ coverage across the approximate $61 \mathrm{Mb}$ genome and over 70,000 single nucleotide polymorphisms (SNPs) were mapped against reference strains. TgCkUg2 was shown to contain entire chromosomes of either type II or type III origin, demonstrating chromosome sorting rather than intrachromosomal recombination. We mapped I,252 novel polymorphisms and clusters of new SNPs within coding sequence implied selective pressure on a number of genes, including surface antigens and rhoptry proteins. Further sequencing of the remaining isolates, six type II and one type III strain, confirmed the presence of novel SNPs, suggesting these are local allelic variants within Ugandan type II strains. In mice, the type III isolate had parasite burdens at least 30 -fold higher than type II isolates, while the recombinant strain had an intermediate burden.

Conclusions: Our data demonstrate that recombination between clonal lineages does occur in nature but there is nevertheless close homology between African and North American isolates. The quantity of high confidence SNP data generated in this study and the availability of the putative parental strains to this natural recombinant provide an excellent basis for future studies of the genetic divergence and of genotype-phenotype relationships.
\end{abstract}




\section{Background}

Toxoplasma gondii is a ubiquitous protozoan parasite of medical and veterinary importance. It can be transmitted via vertical transmission, through carnivory and by ingestion of highly infectious oocysts excreted by the definitive felid hosts [1]. Despite its worldwide distribution, broad host range and multiple transmission routes, which give ample opportunities for strain partitioning and recombination, T. gondii has an unusual population structure dominated by a limited number of clonal lineages [2]. Experimental crosses have shown that a single mating opportunity between two strains in the definitive host can result in a multitude of new strains with altered phenotypic properties [3-6], yet this appears to be rare in nature and only three clonal strain types, called I, II and III, predominate across Europe and North America $[7,8]$. As data become available from wider geographical studies it is evident that higher levels of allelic variation and clonal expansion of non-archetypal lineages occur in South America [911]. While the global population structure may be more complex than previously thought, classification of strains into types I, II and III is still highly relevant in Europe, North America and possibly also in Africa [12].

The three lineages are believed to originate from a few crosses between closely related ancestral strains and generally show a biallelic single nucleotide polymorphism (SNP) pattern where, for any given chromosomal region, two out of the three strains share one allele while the third strain is different [7]. Only one chromosome (Ia) is virtually monomorphic among the three lineages and one chromosome (IV) is dominated by type III SNPs, while all the other chromosomes have a predominance of either type I or type II SNPs or display a chimeric SNP pattern [13]. The full genome sequences of one reference isolate from each of the three clonal lineages have been generated and are available through the Toxoplasma genome database, ToxoDB $[14,15]$, and this detailed information has been used to reconstruct the deep evolutionary relationships between lineages [13,16]. Estimates of within lineage variation have also been made, with the focus on mapping the biogeographical distribution of strain haplotypes to infer patterns of dispersal and disease spread [12,17]. These studies are based on sequence analysis of selected loci from multiple strains, but no comparison has ever been made between two isolates from the same lineage at the genome level. In an environment where strains from a single lineage dominate, it becomes important to estimate the level of allelic variation as recombination may mainly be between strains of the same type.

Recent studies have found evidence of clonal types I, II and III in Africa $[18,19]$, a continent with a wide range of diverse habitats and, like South America, many felid species. Our previous study [19] identified mixed infections in five out of twenty free-range chickens (Gallus domesticus). The presence of multiple strains in a single intermediate host increases the likelihood of recombination between genotypes.
Initial analysis of isolates from this source led to the identification of a putative natural recombinant strain. In this study, we report the whole genome sequencing of this isolate, TgCkUg2, a recombinant between type II and III. Alignment with the reference genomes for Me49 (type II) and VEG (type III), revealed which parts of the genome were inherited from the respective parental strains, and, furthermore, allowed us to look for intralineage divergence and discover new polymorphisms. Comparisons with additional isolates from the same source, one with type III and six with type II alleles, enabled detection of local allelic variants and preliminary genotype-phenotype associations. This is the first whole genome sequencing of a recombinant $T$. gondii strain and the quality of information generated and availability of the putative parental strains to this natural recombinant provide an excellent basis for a better understanding of the gene combinations responsible for virulence and successful transmission of $T$. gondii.

\section{Results}

\section{Sequencing and SNP mapping in TgCkUg2}

Preliminary genotyping led to the conclusion that one of our eight Ugandan isolates contained loci typical of both type II and type III strains and was therefore likely to be a natural recombinant. To gain more information on the nature of the recombination event and on the relationship between the reference strain types and this isolate, TgCkUg2 was subjected to whole genome sequencing and SNP mapping using the 454 Life Sciences platform. Three runs were performed, generating approximately fourfold coverage. We assembled 673,878 reads into 67,013 contigs, ranging from 95 to $12,769 \mathrm{bp}$ with an average length of $773 \mathrm{bp}$. The contigs were aligned against the complete genome sequence of the Me49 reference strain using version 4.3 of the Toxoplasma database [14] and found to span $51.84 \mathrm{Mb}$, corresponding to a genome coverage of $84 \%$ (full details of data deposition are given in Materials and methods). There was no particular bias between the 14 chromosomes in terms of the read density or contig coverage. The data generated for all chromosomes are summarised in Table 1.

To determine the relative contribution of type II and type III regions to the recombinant isolate, the genome of TgCkUg2 was compared to Me49 (II) and VEG (III). SNPs were defined based on $100 \%$ concordance over a minimum of three reads, of which at least one was in the forward direction and one in the reverse. The total number of unambiguous SNPs identified using these stringent criteria and excluding repeat regions was 72,746 , which corresponds to about a quarter of the > 300,000 known polymorphisms between Me49 and VEG. Most SNPs were mapped against either Me49 or VEG, so that TgCkUg2 had the same allele as one of the reference strains, reflecting the origin of this chromosomal region. In addition, 1,252 novel polymorphisms were found where TgCkUg2 was different from both Me49 and VEG. The distri- 
Table I

\begin{tabular}{|c|c|c|c|c|c|c|c|c|c|c|}
\hline Number & $\begin{array}{r}\text { Chromoso } \\
\text { me length } \\
\text { (bp) }\end{array}$ & $\begin{array}{l}\text { Number } \\
\text { of reads* }\end{array}$ & $\begin{array}{r}\text { Total } \\
\text { contig } \\
\text { length (bp) }\end{array}$ & $\begin{array}{l}\text { Coverage } \\
\text { by contigs }\end{array}$ & $\begin{array}{l}\text { Average } \\
\text { reads/kb }\end{array}$ & $\begin{array}{r}\text { Type II } \\
\text { SNPs* } \\
\text { (Greent) }\end{array}$ & $\begin{array}{r}\text { Type III } \\
\text { SNPs } \ddagger \\
\text { (Bluet) }\end{array}$ & $\begin{array}{r}\text { Novel } \\
\text { SNPs§} \\
\text { (Oranget) }\end{array}$ & $\begin{array}{r}\text { Major } \\
\text { SNPI } \\
\text { density/kb }\end{array}$ & $\begin{array}{r}\text { Minor } \\
\text { SNP } ¥ \\
\text { density/kb }\end{array}$ \\
\hline la & I,896,408 & 20,649 & I,585, I 40 & $83.59 \%$ & 13.03 & 2 & 128 & 7 & 0.067 & 0.005 \\
\hline $\mathrm{lb}$ & $1,956,324$ & 20,583 & $1,639,876$ & $83.82 \%$ & 12.55 & I & 1,483 & 10 & 0.758 & 0.006 \\
\hline II & $2,302,931$ & 24,968 & $1,939,495$ & $84.22 \%$ & 12.87 & 4,370 & 73 & 52 & 1.898 & 0.054 \\
\hline III & $2,470,845$ & $26,77 \mid$ & $2,060,909$ & $83.41 \%$ & 12.99 & 27 & 4,224 & 73 & 1.710 & 0.040 \\
\hline IV & $2,576,468$ & 27,510 & $2,150,897$ & $83.48 \%$ & 12.79 & 4,731 & 176 & 60 & 1.836 & 0.092 \\
\hline V & $3,147,601$ & 33,619 & $2,582,080$ & $82.03 \%$ & 13.02 & 54 & 5,725 & 26 & 1.819 & 0.025 \\
\hline VI & $3,600,655$ & 39,723 & $3,042,491$ & $84.50 \%$ & 13.06 & 1,985 & 168 & 99 & 0.551 & 0.074 \\
\hline VIlla & $4,502,211$ & 48,365 & $3,797,608$ & $84.35 \%$ & 12.74 & 8,663 & 79 & 56 & 1.924 & 0.030 \\
\hline VIlb & $5,023,822$ & 53,768 & $4,231,65 I$ & $84.23 \%$ & $12.7 \mid$ & 62 & 5,530 & 41 & 1.101 & 0.021 \\
\hline VIII & $6,923,375$ & 75,308 & $5,851,305$ & $84.52 \%$ & 12.87 & 123 & 6,775 & 158 & 0.979 & 0.041 \\
\hline IX & $6,384,456$ & 72,298 & $5,337,365$ & $83.60 \%$ & 13.55 & 8,119 & 325 & 269 & 1.272 & 0.093 \\
\hline$x$ & $7,418,475$ & 85,205 & $6,298,377$ & $84.90 \%$ & 13.53 & 13,459 & 236 & 209 & 1.814 & 0.060 \\
\hline$X I$ & $6,570,290$ & 70,653 & $5,549,592$ & $84.46 \%$ & 12.73 & 49 & 60 & 117 & - & 0.034 \\
\hline XII & $6,871,637$ & 74,458 & $5,770,139$ & $83.97 \%$ & 12.90 & 58 & 4,809 & 75 & 0.700 & 0.019 \\
\hline Total & $61,645,498$ & 673,878 & $51,836,925$ & $84.09 \%$ & 13.00 & 41,703 & $29,79 \mid$ & I,252 & 1.264 & 0.042 \\
\hline
\end{tabular}

*SNPs denoting a type II background. TgCkUg2 is identical to Me49, but different from VEG. TThe colors correspond to the colours used for the respective SNP types in Figures I and 2 and Additional data files I and 2. \$SNPs denoting a type III background. TgCkUg2 is identical to VEG, but different from Me49. §SNPs where TgCkUg2 has a novel allele, different from both Me49 and VEG. IThe predominant SNP type, corresponding to the chromosomal background type. ${ }^{*} S N P s$ where TgCkUg2 differs from the background type, including novel SNPs.

bution of SNPs called against the two reference strains was highly disproportionate (Figure 1; Additional data file 1) and indicated the genotype of each chromosome. Chromosomes II, IV, VI, VIIa, IX and X were inherited from a type II-like strain while chromosomes Ia, Ib, III, V, VIIb, VIII and XII originated from the type III-like parent.

Due to the paucity of SNPs between types II and III on chromosome XI, it was difficult to derive its source of origin. In total, 226 SNPs were called over its full length of > 6.5 Mb, which averages one SNP per $29 \mathrm{~kb}$. Most of these (117 SNPs) were unique to TgCkUg2, while 49 positions were identical to Me49 and 60 to VEG. There was no obvious clustering of SNPs according to strain on this chromosome and, therefore, no evidence of chromosomal recombination. In total, across all chromosomes, there was a nearly equal contribution from both parental strains to the genome of TgCkUg2, which is consistent with a single sexual reproduction event. Six type II and seven type III chromosomes encompassing 26.8 and 28.3 $\mathrm{Mb}$, respectively, were found, plus one chromosome that might derive from either parent.

Seven chromosomes (Ib, III, VI, VIIb, VIII, IX and XII) showed dramatic changes in the density of their predominant SNP type across their length (Figure 1; Additional data file 1). The predominant type, or 'major SNP', matches the parental allele and corresponds to the divergence between lineages II and III, while the term 'minor SNP' is used for SNPs that do not match the background type of the chromosome. Absence of major SNPs signifies a high level of similarity between types II and III, which corresponds to regions dominated by type I SNPs in the comparison between the three reference strains (where biallelic SNPs are named by the diverging genotype [13]).

Data from the three reference type strains were used to map the relative abundance of type I, II and III SNPs across the parasite genome. Comparison of SNPs from the recombinant strain against this distribution demonstrated that all regions without major SNPs in TgCkUg2 corresponded to the regions dominated by type I SNPs (Figure 2; Additional data file 2). The close matching of these independently retrieved data sets provides strong evidence that TgCkUg2 is the progeny of a cross between modern type II and III strains, where chromosome sorting was the main mechanism of recombination.

\section{The apicoplast}

Most of the apicoplast genome (> 71\%) was covered by a single large contig of $25 \mathrm{~kb}$. The read density was considerably higher than the average read density for the chromosomal contigs: 121.5 reads/kb for the apicoplast compared with 12.55 to 13.55 reads/ $\mathrm{kb}$ for the chromosomal regions (Table 1). The unbiased mechanism of 454 sequencing results in automatic quantification of amplified regions [20], and the higher read density thus implies an average apicoplast genome copy number of nine or ten. This result is slightly higher than the 5 to 7 copies reported initially [21,22], but lower than the $>25$ copies suggested later [23], which could 


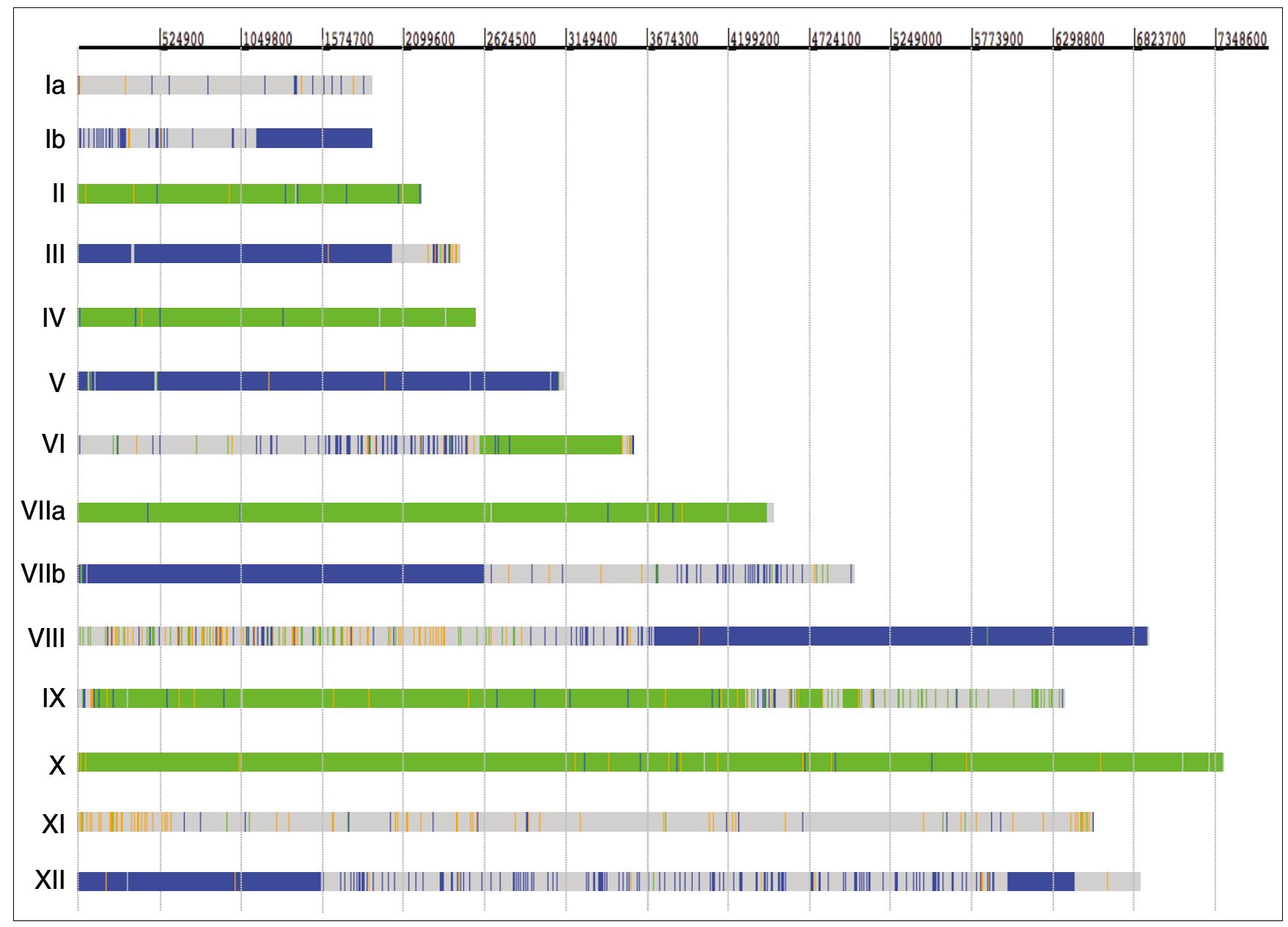

\section{Figure I}

SNP distribution in TgCkUg2. The genomic sequence of TgCkUg2 was aligned with the sequences of Me49 and VEG [I4]; the SNP distribution over the I4 chromosomes is shown above. Green SNPs denote a type II background, where TgCkUg2 is identical to Me49, but different from VEG. Blue SNPs denote a type III background, where TgCkUg2 is identical to VEG, but different from Me49. Orange indicates novel SNPs where TgCkUg2 is different from both reference strains. Grey areas are devoid of SNPs between these strains; genotypes II and III are highly similar in these regions.

be due to inherent differences between strains or methodological differences.

The apicoplast sequence currently available in ToxoDB is from RH, a type I strain. Alignment of the apicoplast genomes of TgCkUg2 and RH resulted in 23 SNP calls over 25,069 bp of sequence. The sequence surrounding each of these SNPs was BLASTed against the sequence data from Me49, VEG and GT1 in the NCBI Trace Archive. Out of 23 high-confidence SNPs detected between TgCkUg2 and RH, all positions were identical in TgCkUg2, Me49 and VEG, while the comparison to GT1 showed three discrepancies. The apicoplast is inherited from the macrogamete in a cross [24], but due to the high level of similarity between types II and III and the fragmented nature of the data in the NCBI Trace archive, it was not possible to ascertain the maternal inheritance of the recombinant strain.

\section{Novel SNPs}

In the alignments of the TgCkUg2 genome with Me49 and VEG, 1,252 positions were found where the two reference strains were identical but the Ugandan strain TgCkUg2 had a different allele. However, based on the SNP discovery rate with the coverage and cut-off criteria we used, the real density of novel SNPs is likely to be around four times higher. The new SNPs were dispersed across all chromosomes, and they occurred at an average frequency of one per $50 \mathrm{~kb}$, but at a higher frequency in the subtelomeric regions of chromosomes (terminal 10\%). In total, $38.1 \%$ of novel SNPs were found in these regions compared with $21.4 \%$ of all SNPs, and this difference was highly significant $(P<0.001$, chi-square test). Several chromosomes had one, or a few, clusters with a high level of new mutations and smaller clusters were found on all chromosomes except Ia. The highest concentrations of new SNPs were found in the subtelomeric regions of chromo- 


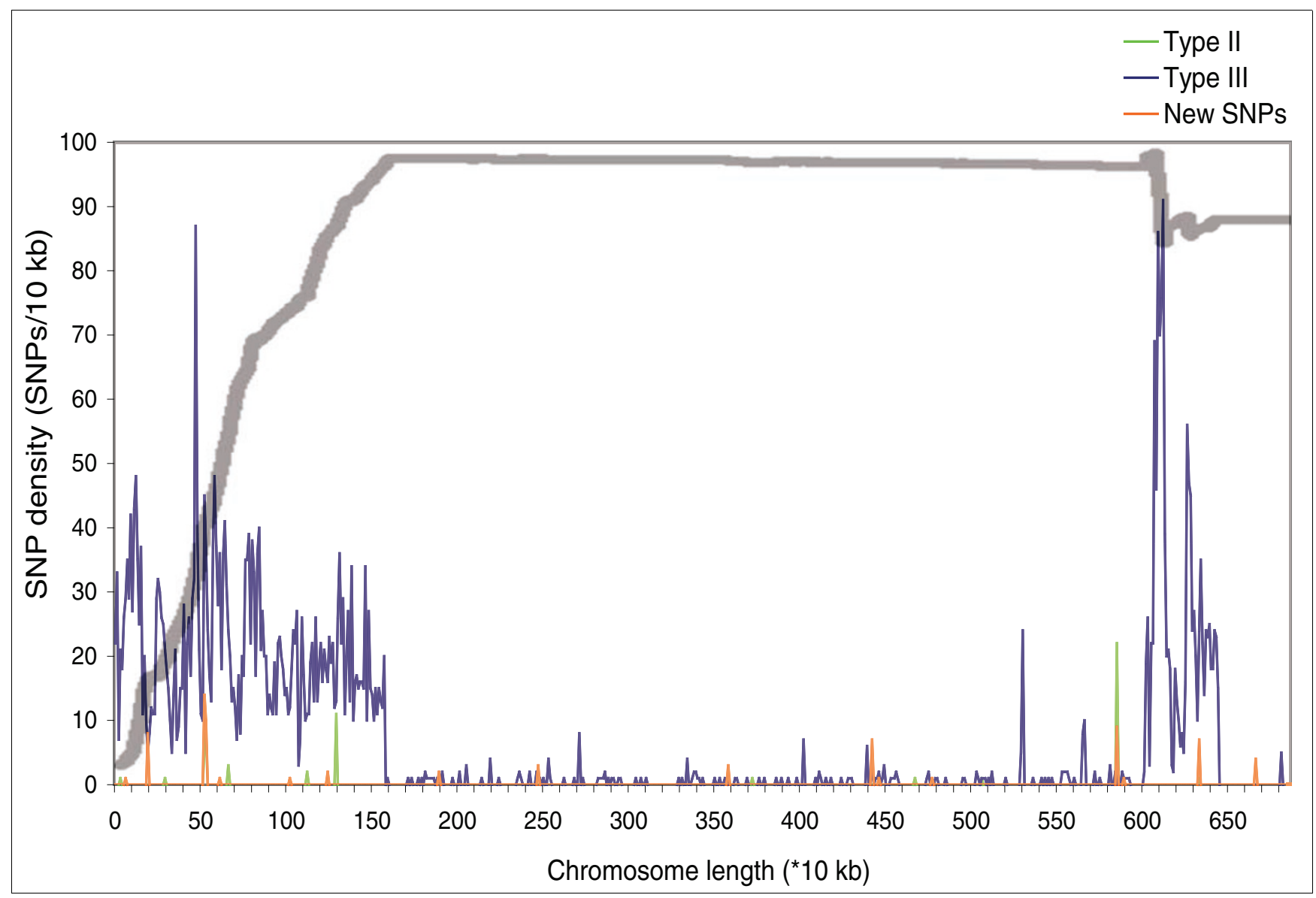

Figure 2

Matching of type I SNP dominance and regions with a low SNP density in TgCkUg2. Comparison of SNP patterns in TgCkUg2 with those in the three sequenced reference genomes, GTI (I), Me49 (II) and VEG (III) on chromosome XII. The underlying graph depicts the SNPs in TgCkUg2 relative to the type II and III reference strains (green and blue, respectively) as well as those unique to TgCkUg2 (orange). In TgCkUg2, chromosome XII was derived from the type III parent (as shown by a predominance of TgCkUg2 SNPs that matched the reference type III at that position), but had large regions with a very low SNP content. To correlate these regions of high and low polymorphism content with existing polymorphism data, all type I, II and III SNPs derived from the reference genome sequences were obtained. For each identified SNP, a running sum was computed across the chromosome as follows: +0 for a type I SNP, + I for a type II SNP, and - I for a type III SNP. This running sum was then plotted against the position in the genome of that SNP, creating the grey line shown. This shows that for the first I.5 Mb of chromosome XII, type II SNPs predominate in the reference strains (types I, II and III, as indicated by the rising line), but at these positions TgCkUg2 has the type III allele (as indicated by the blue line). From approximately I.5 Mb to $6 \mathrm{Mb}$, the chromosome is dominated by type I SNPs in the reference strains (as indicated by the straight grey line) and correspondingly there are very few polymorphisms between TgCkUg2 and the reference strains (similar maps for all 14 chromosomes can be found in Additional data file 2).

somes III, IX and X, and in more central regions of VI and VIII (Figure 3). These novel SNPs occasionally coincided with the allele found in the type I reference strain but most are likely to be the result of new mutations. However, within a short region encompassing $103 \mathrm{bp}$ on chromosome IV, 16 SNPs were found where TgCkUg2, and several of the other Ugandan type II strains, were similar to GT1, but different from VEG and Me49 (Table 2). This similarity only applied to a short region near the chromosomal end and could be a remnant of an earlier recombination event.

Novel SNPs were assigned as non-coding, synonymous or non-synonymous based on gene annotations for Me49 from the Toxoplasma genome database [14]. Most of the novel polymorphisms were non-coding mutations in intergenic or intronic regions, but among the coding SNPs there were twice as many non-synonymous as synonymous mutations, 111 and 55, respectively. Fifteen genes were identified that had at least two novel SNPs in the coding sequence of TgCkUg2 and these are listed in Table 3. Nine of these had a predominance of non-synonymous SNPs and three genes contained six or more mutations that resulted in amino acid substitutions: genes 2.mooo67 and 49.mo3279, which are currently annotated as hypothetical proteins in ToxoDB, and gene 551. moo238 on chromosome XII, which encodes the secreted rhoptry kinase $\mathrm{ROP}_{5}$. 
Table 2

Polymorphisms on chromosome IV, positions 8,805 to 8,907 , where TgCkUg2 and several Ugandan type II strains shared allelic variants with GTI (type I)

Locus $\quad$ IV-8

\begin{tabular}{|c|c|c|c|c|c|c|c|c|c|c|c|c|c|c|c|c|}
\hline Me49 (II) & $\mathbf{T}$ & C & $\mathbf{T}$ & $\mathbf{A}$ & $\mathbf{T}$ & $\mathbf{T}$ & $\mathbf{G}$ & G & $\mathbf{A}$ & $\mathbf{G}$ & $\mathbf{G}$ & G & $\mathbf{A}$ & $\mathbf{A}$ & G & $\mathbf{T}$ \\
\hline VEG (III) & $*$ & $*$ & $*$ & $*$ & $*$ & $*$ & $*$ & $*$ & $*$ & $*$ & $*$ & $*$ & $*$ & $*$ & $*$ & $*$ \\
\hline $\mathrm{TgCkUg6}$ & $*$ & $*$ & * & $*$ & $*$ & $*$ & $*$ & $*$ & $*$ & $*$ & $*$ & $*$ & $*$ & * & $*$ & $*$ \\
\hline TgCkUg5 & $*$ & $*$ & $*$ & $*$ & $*$ & $*$ & $*$ & $*$ & $*$ & $*$ & $*$ & $*$ & $*$ & $*$ & * & $*$ \\
\hline TgCkUg9 & $*$ & $*$ & $*$ & $*$ & $*$ & $*$ & $*$ & $*$ & * & $*$ & $*$ & $*$ & $*$ & $*$ & $*$ & $*$ \\
\hline TgCkUg7 & $*$ & $*$ & A & $\mathrm{T}$ & C & C & C & $*$ & * & $\mathrm{T}$ & $*$ & $*$ & G & $\mathrm{C}$ & C & $*$ \\
\hline $\mathrm{TgCkUgI}$ & C & A & A & $\mathrm{T}$ & $\mathrm{C}$ & C & C & C & $C$ & $\mathrm{~T}$ & A & $A$ & G & $\mathrm{C}$ & $C$ & A \\
\hline $\mathrm{TgCkUg} 2$ & C & $A$ & A & $\mathrm{T}$ & C & $C$ & C & C & $C$ & $\mathrm{~T}$ & A & $A$ & G & C & $C$ & A \\
\hline $\mathrm{TgCkUg3}$ & C & $A$ & A & $\mathrm{T}$ & $\mathrm{C}$ & $C$ & C & C & $C$ & $\mathrm{~T}$ & $A$ & $A$ & G & $\mathrm{C}$ & $C$ & A \\
\hline TgCkUg8 & C & A & A & $\mathrm{T}$ & $C$ & $C$ & C & C & $C$ & $\mathrm{~T}$ & A & $A$ & G & $C$ & $C$ & A \\
\hline GT I (I) & C & A & $\mathbf{A}$ & $\mathbf{T}$ & C & C & C & C & C & $\mathbf{T}$ & $\mathbf{A}$ & $\mathbf{A}$ & G & C & C & $\mathbf{A}$ \\
\hline
\end{tabular}

Similarities to the Me49 sequence are indicated by an asterisk.

In order to detect genes under selection, we used the whole genome sequences from Me49, VEG and TgCkUg2 and performed maximum likelihood pairwise comparisons between all genes to calculate the ratio of non-synonymous to synonymous mutations $(\mathrm{dN} / \mathrm{dS})$. This was followed by a likelihood ratio test to select genes that had a $\mathrm{dN} / \mathrm{dS}$ ratio significantly $(P<0.05)$ higher than one [25]. Using these criteria, evidence for selection was detected for 46 genes (Additional data file 3 ). These candidates included genes encoding four dense granule proteins, GRA3 (42.mooo13), GRA6 (63.moooo2), GRA7 (20.moooo5) and GRA8 (52.moooo2), the rhoptry kinase family protein $\mathrm{ROP} 4 / 7 \quad(83 . \mathrm{mo2145})$ and the bradyzoite surface protein SRS16B (641.m01562), previously identified in the analysis of novel SNP clusters (Table 3). A

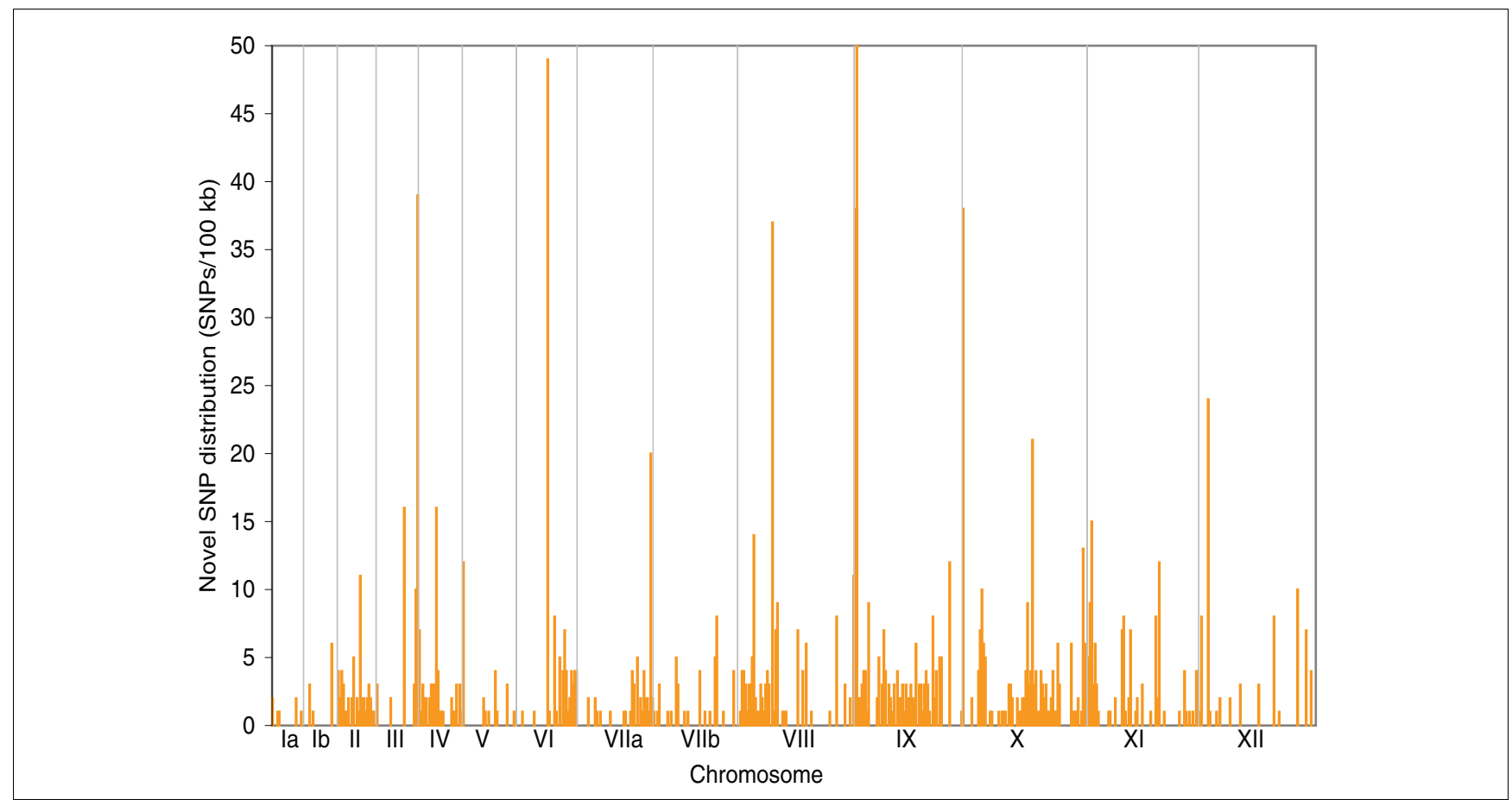

Figure 3

Location and density of unique SNPs in TgCkUg2. The graph shows the number of SNPs per $100 \mathrm{~kb}$, where $\mathrm{TgCkUg2}$ had a different allele compared with Me49 and VEG. New SNPs were distributed across the whole genome, but very high densities were found near the telomeres of chromosomes III, VIla, IX and X, but also in central regions of chromosomes VI and VIII. These mutation hot-spots were mostly located in intergenic regions, but also caused a high number of mutations in the genes for hypothetical proteins 2.m00067, $42 . \mathrm{m} 07434$ and the rhoptry antigen ROP5 (55I.m00238). 
Table 3

\begin{tabular}{|c|c|c|c|c|c|c|}
\hline \multirow[t]{2}{*}{ Gene ID (v4.3)* } & \multirow[b]{2}{*}{ Number } & \multicolumn{2}{|c|}{ Novel SNPs in TgCkUg2 } & \multicolumn{2}{|c|}{ SNPs between the three lineages ${ }^{\dagger}$} & \multirow[b]{2}{*}{ Protein description } \\
\hline & & Synonymous & Non-synonymous & Synonymous & Non-synonymous & \\
\hline $641 . \mathrm{m} 01562$ & IV & I & I & 12 & 86 & SRSI6B \\
\hline $641 . m 02553$ & IV & 1 & I & 6 & I & WD-40 repeat protein, putative \\
\hline 49.m03276 & $\mathrm{VI}$ & 1 & I & 0 & 4 & ROP29 \\
\hline 49.m03279 & $\mathrm{VI}$ & 2 & 8 & I & 13 & Hypothetical \\
\hline 49.m03372 & VI & 1 & I & 13 & 3 & Long chain fatty acid CoA ligase \\
\hline 55.m04829 & VIlb & 1 & 3 & I & 2 & SRS26A \\
\hline 44.m02583 & VIII & 0 & 2 & 9 & 11 & Hypothetical \\
\hline 44.m05903 & VIII & 0 & 2 & 7 & 8 & Hypothetical \\
\hline 57.m0I765 & IX & 0 & 2 & 143 & 231 & Protein kinase domain containing \\
\hline 2.m00067 & IX & 3 & 7 & 0 & 0 & Hypothetical \\
\hline 57.m0I732 & IX & 0 & 2 & 7 & 8 & Hypothetical \\
\hline 80.m02252 & IX & 1 & I & 4 & 2 & Phosphoenolpyruvate carboxykinase, putative \\
\hline 42.m07434 & $x$ & 0 & 2 & 0 & 0 & Hypothetical \\
\hline $551 . m 00238$ & XII & 3 & 6 & 8 & 44 & ROP5 \\
\hline $65 . \mathrm{m} 0000 \mathrm{I}$ & XII & 4 & 0 & 9 & 6 & NTPase I \\
\hline
\end{tabular}

*Since the comparison was made against the annotation of Me49 in v4.3 of ToxoDB, these gene IDs are used throughout. These remain searchable in the current annotation ( $v 5.0$ ). tData from ToxoDB showing the total number of SNPs between GTI, Me49 and VEG, in order to put the number of novel SNPs into context.

subset of 16 genes had very high $\mathrm{dN} / \mathrm{dS}$ values, indicating that they may be under positive selection in TgCkUg2 (Table 4). These included the genes encoding GRA3 and ROP4/7 and 551.mo0237, a gene immediately adjacent to that encoding $\mathrm{ROP}_{5}$. One gene (42.mo7434) located on chromosome $\mathrm{X}$ exhibited significant divergence between TgCkUg2 and its chromosomal background genotype. This is currently annotated as a hypothetical protein and nothing is known about its function or localization.

Estimated divergence of African and reference isolates SNP data from TgCkUg2 were used to estimate the age of the most recent common ancestor (MRCA) of the Ugandan types II and III (UgII and UgIII) and the reference strains of the respective types. Six type II chromosomes of this recombinant strain were used for the Me49/UgII calculations and seven chromosomes of type III origin were used to estimate the VEG/UgIII split. Calculations are shown for all chromosomes separately as well as for the full type II and type III sequences using two different approaches (Tables 5 and 6).

The estimated T. gondii intron mutation rate of $1.94 \times 10^{-8}$ mutations per nucleotide per year [26] was applied to minor SNPs found in intronic regions across the genome. This was achieved by retrieving all SNPs where TgCkUg2 was different from Me49 within the introns of type II chromosomes (II, IV, VI, VIIa, IX and X), and similarly all SNPs where TgCkUg2 differed from VEG for type III chromosomes (Ia, Ib, III, V, VIIb, VIII and XII). In total, the type II intronic regions contained 381 minor SNPs over 1.13 Mb, which gives an estimate of 17,400 years for the MRCA of UgII and Me49. The type III regions contained 229 SNPs over 1.28 Mb, giving an estimate for the divergence of UgIII and VEG of 9,200 years. Substantial variation was found between chromosomes from the same lineage; however, all type II chromosomes except VIIa gave earlier divergence time estimates than chromosomes of type III.

The second method related data on major and minor SNPs, where major SNPs were assumed to represent the divergence between types II and III at the nucleotide level based on an estimated MRCA at 150,00o years ago [27], while minor SNPs were assumed to represent the intralineage divergence between Ugandan and reference strains. Regions dominated by type I SNPs were excluded from this analysis since they do not contain a major SNP type. These calculations resulted in divergence time estimates, which were considerably more recent; 4,600 years for UgII/Me49 and 1,600 years for UgIII/ VEG. The overall genomic mutation rate was calculated by a weighted average of the type II and III regions and estimated 
Table 4

Genes under selection identified by $\mathrm{dN} / \mathrm{dS}$ analysis in TgCkUg2

\begin{tabular}{|c|c|c|c|c|c|}
\hline Chromosome (type*) & Comparator & Gene ID (v4.3) & Protein description & $\mathrm{dN} / \mathrm{dS}$ ratio ${ }^{\dagger}$ & $P$-value \\
\hline la (III) & Me49 (II) & $83 . \mathrm{m} 02145$ & Rhoptry kinase ROP4/ROP7 & Infinity & $0.025<P<0.050$ \\
\hline IV (II) & VEG (III) & $641 . \mathrm{m} 01516$ & Hypothetical & Infinity & $0.005<P<0.010$ \\
\hline V (III) & Me49 (II) & $39 . \mathrm{m} 00623$ & Proline-rich protein & Infinity & $0.010<P<0.025$ \\
\hline V (III) & Me49 (II) & $31 . \mathrm{m} 01816$ & Iron-sulfur cluster assembly accessory protein, putative & Infinity & $0.010<P<0.025$ \\
\hline V (III) & Me49 (II) & 76. $\mathrm{m0I} 544$ & Hypothetical & Infinity & $0.010<P<0.025$ \\
\hline VI (II) & VEG (III) & 49.m03376 & Hypothetical & Infinity & $0.025<P<0.050$ \\
\hline VI (II) & VEG (III) & 49.m03382 & Hypothetical & Infinity & $0.010<P<0.025$ \\
\hline VI (II) & VEG (III) & 49.m03431 & Hypothetical & Infinity & $0.010<P<0.025$ \\
\hline VIII (III) & Me49 (II) & 59.m07776 & Hypothetical & Infinity & $0.025<P<0.050$ \\
\hline VIII (III) & Me49 (II) & 59.m0336I & Transporter, major facilitator family domain containing & 4.325 & $0.010<P<0.025$ \\
\hline$X(I I)$ & Me49 (II) & 42.m07434 & Hypothetical & Infinity & $0.010<P<0.025$ \\
\hline$X(I I)$ & VEG (III) & 42.m03570 & LytB domain-containing protein & Infinity & $0.025<P<0.050$ \\
\hline$X(I I)$ & VEG (III) & $42 . \mathrm{m} 00013$ & GRA3 & Infinity & $0.010<P<0.025$ \\
\hline X (II) & VEG (III) & 46.m02909 & Hypothetical & Infinity & $0.025<P<0.050$ \\
\hline XII (III) & Me49 (II) & 55I.m00237 & Hypothetical & Infinity & $0.025<P<0.050$ \\
\hline XII (III) & Me49 (II) & |45.m00337 & Hypothetical & 6.527 & $0.010<P<0.025$ \\
\hline
\end{tabular}

*The chromosomal background genotype. TThe number of non-synonymous SNPs divided by the number of synonymous SNPs. In most cases, no synonymous SNPs were found, and this rate approaches infinity.

to be approximately $1.28 \times 10^{-8}$ mutations per nucleotide per year, which corresponds to $66 \%$ of the rate calculated for intronic regions.

Finally, an estimate of the age of the MRCA of all strains was calculated based on major and minor SNPs in intronic regions using the results obtained by application of the intron mutation rate. These estimates were considerably higher than the proposed 150,000 years, suggesting a MRCA about $10^{6}$ years ago, which is similar to the timing proposed for the divergence of South American strains [8]. The data used for these calculations are provided in Additional data file 4.

\section{Relationships between Ugandan isolates}

In addition to TgCkUg2, one type III strain, TgCkUg6, and six type II strains, TgCkUg1, 3, 5, 7, 8 and 9, were isolated from Uganda. We generated and compared sequence data over > $20 \mathrm{~kb}$ across 34 loci (Additional data file 5) distributed across the genome to investigate the genetic relatedness among Ugandan $T$. gondii strains. These loci included known polymorphic genes such as those encoding toxofilin and ROP18, microsatellites and intronic regions. A high level of sequence homology was seen between the novel isolates from Uganda and the reference strains, which originate from North America. The type III strain, TgCkUg6, was very closely related to the type III reference strain VEG as well as the type III regions of TgCkUg2. In comparison to VEG, TgCkUg6 had 39 SNPs over $20.9 \mathrm{~kb}$ and most of these SNPs were concentrated in two loci: II-4 (10 SNPs over 598 bp) and VI-13 (18 SNPs over $368 \mathrm{bp}$ ). Apart from these regions the sequence identity between the type III strains was $>99.9 \%$. Locus II-4 consisted of non-coding subtelomeric sequence on chromosome II, where TgCkUg6 shared some alleles with strains of genotype II (including Me49). The second locus, VI-13, included 220 $\mathrm{bp}$ of the coding sequence of the surface protein SRS22H (49.mo3110), where several new, non-synonymous SNPs were found for TgCkUg6, TgCkUg2 and three of the Ugandan type II strains (Table 7).

The Ugandan type II isolates, including the type II regions of TgCkUg2, were closely related to Me49 (> 99.5\% sequence identity), but with some allelic variation. The new SNPs were largely concentrated at a few loci and many were shared among Ugandan isolates, suggesting that these are local 
Table 5

\begin{tabular}{|c|c|c|c|c|}
\hline Chromosome & Intron length* (bp) & Minor SNPs in introns & MRCA Me49/Ugll (years) & MRCA VEG/UgIII (years) \\
\hline \multicolumn{5}{|l|}{ Type II } \\
\hline ॥ & 89,003 & 34 & 19,691 & \\
\hline IV & 85,471 & 58 & 34,979 & \\
\hline VI & 152,385 & 65 & 21,987 & \\
\hline VIlla & 198,902 & 28 & 7,256 & \\
\hline IX & 266,876 & 117 & 22,598 & \\
\hline$x$ & 334,181 & 79 & 12,186 & \\
\hline Total II & $1,126,818$ & 381 & 17,429 & \\
\hline \multicolumn{5}{|l|}{ Type III } \\
\hline la & 80,522 & 2 & & 1,280 \\
\hline $\mathrm{lb}$ & 85,805 & 8 & & 4,806 \\
\hline III & 82,660 & 9 & & 5,612 \\
\hline V & 119,536 & 15 & & 6,468 \\
\hline VIIb & 220,517 & 39 & & 9,116 \\
\hline VIII & 337,557 & 78 & & $|1,9| \mid$ \\
\hline XII & 352,605 & 78 & & 11,403 \\
\hline Total III & $1,279,202$ & 229 & & 9,228 \\
\hline
\end{tabular}

The divergence between Ugandan and reference strains were calculated based on the intron mutation rate $1.94 \times 10^{-8}[26]$. A more comprehensive version is provided in Additional data file 4. *Length of intronic regions. MRCA, most recent common ancestor.

allelic variants. Interestingly, most new SNPs found within genes, including those encoding toxofilin (33.mo2185) and SRS16B (641.mo1562), resulted in amino acid changes, suggesting they may be under selection. Based on these variants, it was possible to resolve that $\mathrm{TgCkUg} 5$ and $\mathrm{TgCkUg9}$ were the isolates most similar to Me49 and that TgCkUg3 was the strain most similar to the type II component of the recombinant TgCkUg2.

This complementary sequencing confirmed the assignment of TgCkUg2 chromosomes according to 454 SNP analyses, but discovered possible chromosomal recombination events in two type I dominated regions. Chromosome VIII was identified as derived from type III based on the major SNP density in the second half of the chromosome. However, for loci VIII19 and VIII-20 (located at 0.9 and 2.1 Mb) TgCkUg2 was more similar to Me49 than VEG and even contained four allelic variants that were also present in the Ugandan type II isolate TgCkUg8, while locus VIII-21 at 5.8 Mb identified TgCkUg2 as a type III strain (Table 8). Similarly, comparison of TgCkUg2 and TgCkUg6 sequence for the VI-13 locus, located around $0.3 \mathrm{Mb}$, indicated the presence of a type III region in the otherwise type II derived chromosome VI. These results provide indications of chromosomal recombination in TgCkUg2, but the limited extension of the SNP peaks at these locations (Additional data file 1) suggest gene conversions rather than homologous cross-over events.

\section{Phenotype of TgCkUg2 and clonal Ugandan isolates}

None of the Ugandan isolates caused morbidity or mortality in mice and could therefore be classified as avirulent. Quantitative PCR (Q-PCR) of parasite burden in mice used for isolation revealed major differences between strain types (Figure 4). The type III strain TgCkUg6 produced high tissue burdens compared with the Ugandan type II strains, and this difference was significant for brain $(P<0.001)$, heart $(P<0.002)$ and muscle $(P<0.02, t$-test $)$. The type II/III strain (TgCkUg2) caused an intermediate parasite burden for all organs. In brain tissue, the average density estimated via QPCR was $4.5 \times 10^{6}$ parasites per gram for type III, $1.2 \times 10^{6}$ for the recombinant, and $1.5 \times 10^{5}$ for the six type II strains. In heart tissue the mean values for parasite density were $1.2 \times$ $10^{5}$ (III), $8.9 \times 10^{3}$ (II/III) and $6.2 \times 10^{2}(\mathrm{II})$ parasites per gram. The parasite burden caused by the recombinant strain was significantly higher than type II strains $(P<0.003$ for brain, heart and muscle), while the difference between TgCkUg2 and TgCkUg6 did not reach significance. In all mice, the brain was the most heavily infected organ $(P \leq 0.05$, paired $t$-test), and, on average, had more than tenfold higher 
Table 6

\begin{tabular}{|c|c|c|c|c|c|}
\hline Chromosome & Length* (bp) & Minor SNPs & Major SNPs & MRCA Me49/UgIl (years) & MRCA VEG/UgIII (years) \\
\hline \multicolumn{6}{|l|}{ Type II } \\
\hline II & $2,302,931$ & 125 & 4,370 & 4,291 & \\
\hline IV & $2,576,468$ & 236 & 4,731 & 7,483 & \\
\hline $\mathrm{VI}>2.6 \mathrm{Mb}$ & $1,000,655$ & 70 & 1,913 & 5,489 & \\
\hline VIla & $4,502,211$ & 135 & 8,663 & 2,338 & \\
\hline IX 0.5-4 Mb & $3,500,000$ & 205 & 6,349 & 4,843 & \\
\hline$x$ & $7,418,475$ & 445 & 13,459 & 4,960 & \\
\hline Total II & $21,300,740$ & 1,216 & 39,845 & 4,578 & \\
\hline \multicolumn{6}{|l|}{ Type III } \\
\hline $\mathrm{lb}>\mathrm{I} .2 \mathrm{Mb}$ & 756,324 & 6 & 1,375 & & 655 \\
\hline $\mathrm{III}<1.9 \mathrm{Mb}$ & $1,900,000$ & 33 & 3,953 & & 1,252 \\
\hline V & $3,|47,60|$ & 80 & 5,725 & & 2,096 \\
\hline Vllb $<2.5 \mathrm{Mb}$ & $2,500,000$ & 43 & 5,069 & & 1,272 \\
\hline $\mathrm{VIII}>4 \mathrm{Mb}$ & $2,923,375$ & 49 & 6,268 & & 1,173 \\
\hline$X I I<I .5 \mathrm{Mb}$ & $1,500,000$ & 69 & 3,259 & & 3,176 \\
\hline Total III & $12,727,300$ & 280 & 25,648 & & 1,638 \\
\hline
\end{tabular}

The divergence between Ugandan and reference strains were calculated based on their relationship to the divergence between Me49 and VEG, with the MRCA estimated to be at 150,000 years ago [27]. A more comprehensive version is provided in Additional data file 4. *Length of regions with a major SNP type. MRCA, most recent common ancestor.

parasite density than skeletal muscle, 100 times more than heart muscle and 1,000 times more than lung tissue.

Parasite isolates were introduced into culture in human fibroblasts and growth characteristics were assessed by QPCR at passage eight. The growth of all Ugandan isolates was slow in cell culture in comparison to the reference strain
Me49 (Figure 5). This difference was chiefly due to a prolonged lag phase of between 4 and 7 days, which preceded the phase of exponential growth. There was considerable variation between the type II strains, while the type III and the recombinant strain both had intermediate growth rates in vitro. Among the type II isolates, TgCkUg5 and TgCkUg9 were the slowest growing and never reached the parasite den-

Table 7

Local allelic variants in Ugandan T. gondii strains leading to amino acid changes in two surface proteins (SRSs) and one rhoptry protein (toxofilin)

Locus amino acid position

SRS22H (VI-I3)

Toxofilin

SRSI6B

\begin{tabular}{|c|c|c|c|c|c|c|c|c|c|c|c|c|c|c|}
\hline & 111 & 113 & 138 & 139 & 140 & $|4|$ & 143 & 144 & 146 & 150 & 147 & 168 & 176 & 77 \\
\hline Me49 (II) & E & E & $\mathbf{K}$ & $\mathbf{P}$ & $\mathbf{S}$ & A & $\mathbf{H}$ & $\mathbf{R}$ & $\mathbf{T}$ & D & $\mathbf{L}$ & $\mathbf{E}$ & $\mathbf{K}$ & $\mathbf{A}$ \\
\hline TgCkUg5 & $*$ & $\mathrm{G}$ & $*$ & G & $*$ & $*$ & $*$ & $*$ & $*$ & $*$ & $*$ & $*$ & $\mathrm{R}$ & $*$ \\
\hline TgCkUg9 & $*$ & $*$ & $*$ & $*$ & $*$ & $*$ & $*$ & $*$ & $*$ & V & $*$ & $D$ & $\mathrm{R}$ & $*$ \\
\hline TgCkUg7 & $*$ & $*$ & * & $*$ & * & $*$ & $*$ & $*$ & $*$ & $*$ & $\mathrm{Q}$ & $D$ & $\mathrm{R}$ & $\mathrm{E}$ \\
\hline TgCkUgI & $*$ & $*$ & $*$ & $*$ & $*$ & $*$ & $*$ & $*$ & $*$ & $*$ & $\mathrm{Q}$ & $*$ & $*$ & $\mathrm{E}$ \\
\hline TgCkUg8 & $*$ & $*$ & $*$ & $*$ & $*$ & $*$ & $*$ & $*$ & $*$ & $*$ & $\mathrm{Q}$ & $*$ & $*$ & $\mathrm{E}$ \\
\hline $\mathrm{TgCkUg3}$ & $*$ & $*$ & $*$ & $*$ & $*$ & $*$ & $*$ & $*$ & $*$ & $\vee$ & $\mathrm{Q}$ & $*$ & $*$ & $\mathrm{E}$ \\
\hline TgCkUg2 & $\mathrm{D}$ & $\mathrm{G}$ & $\mathrm{T}$ & $\mathrm{G}$ & $\mathrm{T}$ & G & $\mathrm{R}$ & $*$ & $P$ & $\mathrm{~V}$ & $\mathrm{Q}$ & $*$ & $*$ & $\mathrm{E}$ \\
\hline TgCkUg6 & $\mathrm{D}$ & $\mathrm{G}$ & $N$ & G & $*$ & G & $\mathrm{R}$ & $\mathrm{S}$ & $P$ & $\mathrm{~V}$ & $E$ & $*$ & $*$ & $\mathrm{~T}$ \\
\hline VEG (III) & $*$ & $*$ & $*$ & $*$ & $*$ & $*$ & $*$ & $*$ & $*$ & $*$ & $\mathbf{E}$ & $*$ & $*$ & $\mathbf{T}$ \\
\hline GTI (I) & $*$ & $\mathbf{G}$ & $\mathbf{S}$ & $\mathbf{A}$ & $\mathbf{T}$ & E & $\mathbf{R}$ & $\mathbf{S}$ & D & $\mathbf{G}$ & $\mathbf{Q}$ & $*$ & $\mathbf{R}$ & $\mathbf{A}$ \\
\hline
\end{tabular}

Similarities to the reference sequence for Me49 are indicated by an asterisk. 
Table 8

\begin{tabular}{|c|c|c|c|c|c|c|c|c|c|c|c|c|c|c|}
\hline \multirow{2}{*}{$\begin{array}{l}\text { Locus position } \\
\text { Me49 (II) }\end{array}$} & \multicolumn{7}{|c|}{$\begin{array}{l}\text { VIII- } 19 \\
0.9 \mathrm{Mb}\end{array}$} & \multicolumn{4}{|c|}{$\begin{array}{l}\text { VIII-20 } \\
2 . I \mathrm{Mb}\end{array}$} & \multicolumn{3}{|c|}{$\begin{array}{l}\text { VIII-2I } \\
5.8 \mathrm{Mb}\end{array}$} \\
\hline & $\mathbf{A}$ & $\mathbf{T}$ & G & C & $\mathbf{T}$ & $\mathbf{T}$ & $\mathbf{T}$ & C & $\mathbf{T}$ & G & A & G & C & G \\
\hline TgCkUg5 & $*$ & $*$ & $*$ & $*$ & $*$ & $*$ & $*$ & $*$ & $*$ & $*$ & $*$ & $*$ & $*$ & * \\
\hline TgCkUg8 & $*$ & $*$ & $*$ & $*$ & A & C & $*$ & $\mathrm{~T}$ & $*$ & A & $*$ & $*$ & $*$ & $*$ \\
\hline TgCkUg2 & $*$ & $*$ & $*$ & $*$ & A & C & $*$ & $\mathrm{~T}$ & $*$ & A & $*$ & A & A & $\mathrm{T}$ \\
\hline TgCkUg6 & $\mathrm{T}$ & $*$ & C & G & $*$ & $*$ & C & $*$ & C & $*$ & $\mathrm{~T}$ & A & A & $\mathrm{T}$ \\
\hline VEG (III) & $\mathbf{T}$ & C & C & G & * & $*$ & C & $*$ & C & $*$ & $\mathbf{T}$ & A & A & $\mathbf{T}$ \\
\hline
\end{tabular}

For loci VIII-19 and VIII-20 located around 0.9 and $2.1 \mathrm{Mb}$ on chromosome VIII, TgCkUg2 is type II-like and shares some local allelic variants with TgCkUg8. Later on the chromosome, TgCkUg2 is type III-like, as shown by the VIII-2I locus and the major SNP type (Figure I; Additional data file I).

sity levels of the other strains. Figure 5 shows the data for a single passage, but the slower growth of these two strains was consistently observed over several months.

\section{Discussion}

Whole genome sequencing of a natural recombinant T.gondii strain revealed a nearly equal contribution of type II and III alleles, and demonstrated that it is likely to have arisen through a single recombination event between two parental strains. Most chromosomes appeared to have been inherited in their entirety from one parental strain, and the background genotype could easily be deduced from the SNP density. To our knowledge, the genetic combination found in TgCkUg2 has not been detected through multi-locus genotyping elsewhere (C Su, personal communication) nor been observed in progeny from laboratory crosses [28]. Even though novel SNPs were observed in the Ugandan strains, the whole genome sequence of TgCkUg2 showed a high level of sequence identity to the relevant reference strain, either Me49 or VEG for any given region. Certain chromosomal regions had very low SNP densities and these immediately

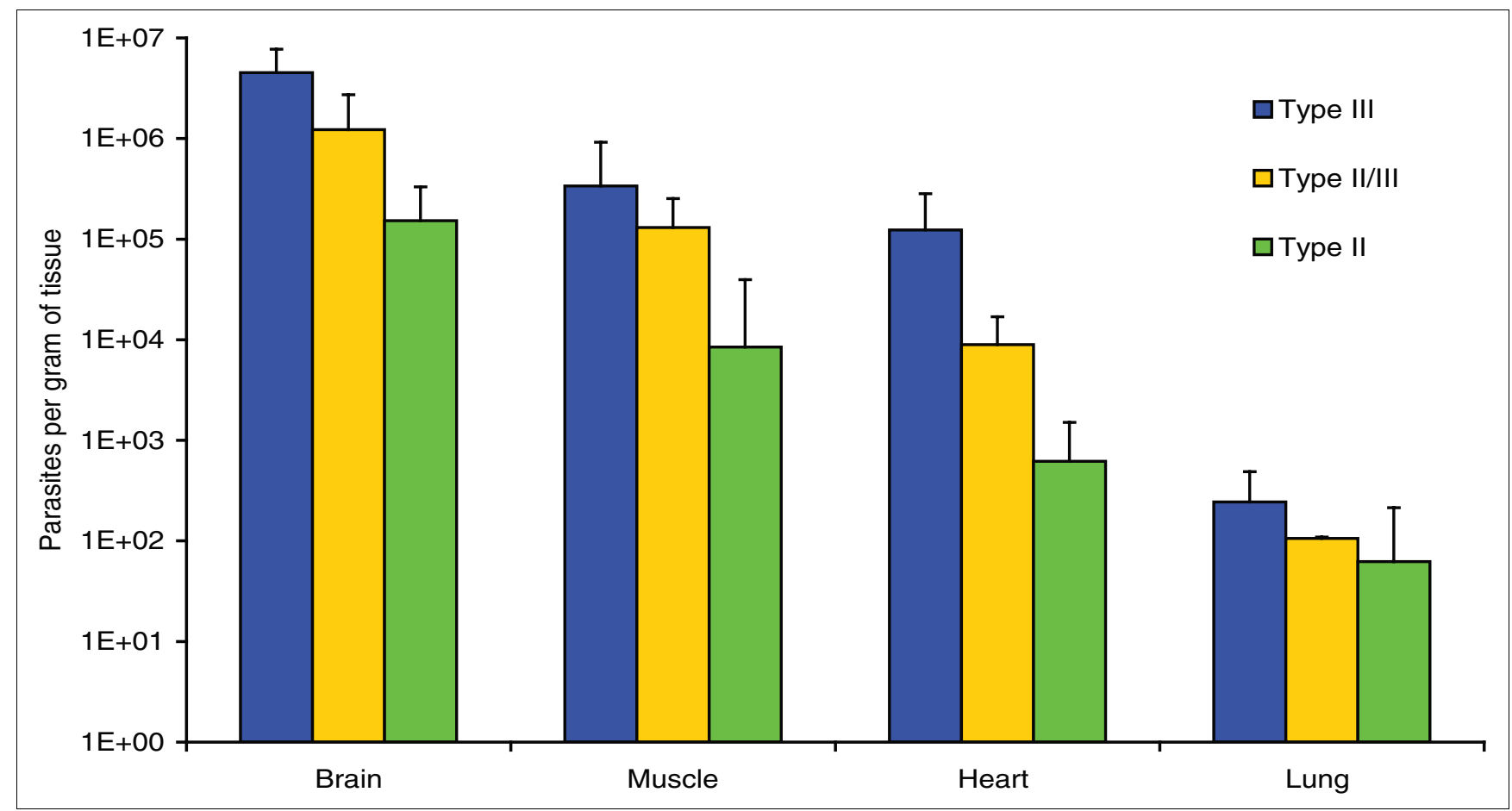

Figure 4

Parasite density in mouse tissues. The parasite burden in mice (number of T. gondii per gram) was determined by quantitative PCR and the graph shows the average values plus standard deviation for one type III strain (three mice), one type II/III strain (two mice) and six type II strains (I7 mice). The type III strain ( $\mathrm{TgCkUg6}$ ) caused the highest burden, followed by the recombinant strain ( $\mathrm{TgCkUg2})$, while the six type II strains $(\mathrm{TgCkUgI}, 3,5,7,8,9)$ had significantly lower densities. In all mice, the brain was the most heavily infected tissue. 


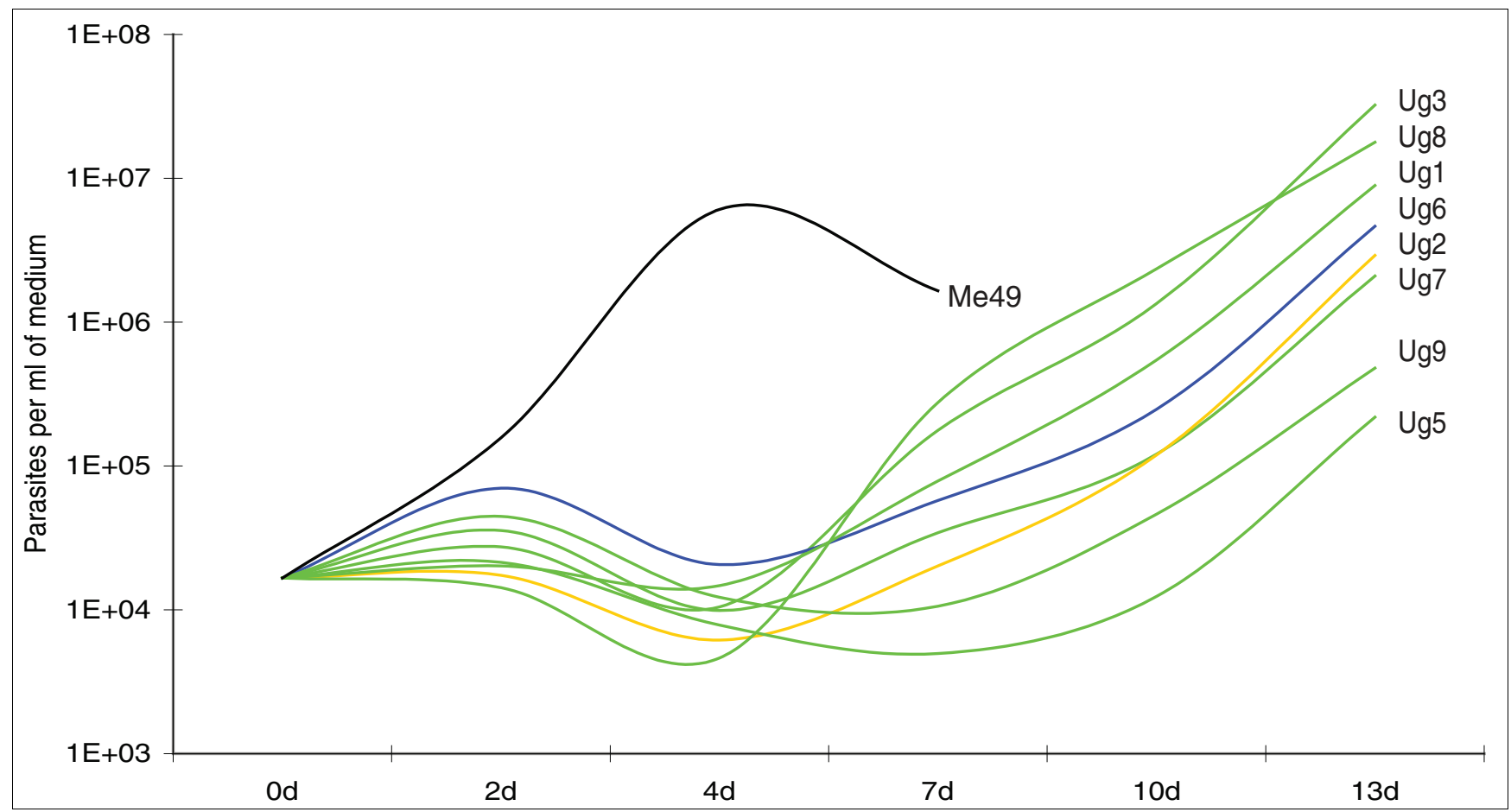

\section{Figure 5}

Number of extracellular parasites per milliliter of medium during growth in cell culture. Q-PCR quantification of free parasites in cell culture (number of $T$. gondii/ml medium) at five different time points ( 0 to 13 days after inoculation) revealed an extended lag phase in all the Ugandan isolates compared with Me49. $\mathrm{TgCkUg6}$ (blue) and $\mathrm{TgCkUg2}$ (orange) had intermediate growth rates, and the fastest growing Ugandan strains were $\mathrm{TgCkUgI}, 3$ and 8, while $\mathrm{TgCkUg} 5$ and 9 were extremely slow growing and never reached high numbers of free parasites. The difference between the Ugandan isolates and Me49 is probably due to their recent introduction in cell culture, but the difference between the Ugandan type II strains may have a genetic component. Interestingly, $\mathrm{TgCkUg5}$ and 9 were the strains with the lowest frequency of local allelic variants.

appeared to coincide with the predominance of type I SNPs [13], which was confirmed by detailed comparisons across the genome (Additional data file 2). The sequencing of this recombinant isolate does not provide evidence of exotic or ancient strains in Uganda, but demonstrates sexual recombination through chromosome sorting between two strains that are closely related to the clonal lineages predominant in Europe and North America.

Sexual recombination between different strains of $T$. gondii appears to be an important evolutionary force, but has been assumed to be a rare event in nature [7]. For recombination to occur more than one genotype must be present in the environment and these must be ingested by the definitive host within a short time frame. The most plausible way for this to happen is for a cat to eat a host animal that is infected by two different strains, but the strong immune response elicited by the primary infection may prohibit the establishment of a second infection in some host species [29,30]. However, the possibility that multiple infections may occur in certain hosts has been proven in laboratory mice [31-33] and is also reported in naturally acquired infections in humans and animals [34-36], including the current strains from Uganda [19]. However, the relative abundance of the different strains in the host tissues may vary considerably and if one strain predominates, selffertilization is likely to be more frequent than recombination between strains. In our Ugandan strains, we found that the difference in parasite burden in mice was up to 1,00o-fold. In the event of multiple infections it is likely that only the most abundant strain would prevail. Indeed, some of our current isolates were obtained from mice inoculated with tissue containing more than one T. gondii genotype [19] but following passage in mice only one strain was recovered. Although there appear to be many obstacles to a successful recombination event, it is likely to be more frequent in environments where multiple parasite strains circulate within an area with close contact between cats and intermediate hosts [35,37].

Analysis of progeny from three experimental crosses (two II $\times$ III $[5,6]$ and one I $\times$ III $[38]$ ) have shown that strains of different genotypes readily recombine to create progeny with predicted levels of exchange. On average, recombinant progeny had three to four recombinant chromosomes; however, one strain had as many as 10 recombinant chromosomes and 5 out of 75 strains $(6.7 \%)$ had none [3,28]. Investigations of many more natural recombinant isolates are required to discover which gene combinations generate successful parasites and whether relative absence of hybrid chromosomes is ben- 
eficial and more common in nature than expected from the frequencies reported in the experimental crosses $[3,5,6,38]$.

Although there was limited genetic divergence between the Ugandan strains and the type reference strains, SNPs against the background type were useful for estimation of the intralineage divergence and for detection of genes under selection. Divergence time estimates rely on the assumption that the mutation rate in a species remains relatively constant over time [39,40]. Without fossil records, calibration of the clock is difficult and in an organism with a complex life cycle, calculation of the mutation rate may be difficult. It is perhaps not surprising that the two methods, which use different data sets and start with different assumptions, give diverging time estimates. The relative timing, however, consistently indicated that the Ugandan type II strains are more divergent from Me49 than the Ugandan type III is from VEG. The estimated times of MRCA using the intronic mutation rate were in the region of $10^{4}$ years, more or less corresponding to the timing of the proposed clonal expansion $[17,26]$. Interestingly, chromosome VIIa had a higher level of similarity with Me49 than the other type II chromosomes and it may originate from a strain that diverged at a later time point. Although limited data exist for the other Ugandan strains, the slow-growing TgCkUg5 and TgCkUg9 seem to be more closely related to Me49 then the other Ugandan type II strains are. In conclusion, these data point towards a partial separation between the different type II populations starting around $10^{4}$ years ago, but genetic exchange may occasionally have occurred between the groups, to give rise to the strains we see today, where different chromosomes have a different level of divergence.

Genome scale analysis of natural recombinant and atypical isolates provide a good tool for mapping genes important for survival and adaptation to different environments. The sequencing of TgCkUg2 generated over 1,200 new SNPs, which were non-randomly distributed in the genome and had a dN/dS ratio greater than 2, suggesting significant selective pressure. Pathogenicity in T. gondii is a multigenic trait and a number of loci and genes associated with virulence have been identified, including those encoding rhoptry proteins (ROPs), dense granule proteins (GRAs) and SAG1 related surface antigens (SRSs) [41-45]. In this study, the recombinant type II/ III strain caused significantly higher tissue burdens in mice compared with the related type II isolates, but it did not reach the elevated parasite densities of the type III isolate. This intermediate phenotype in vivo may be explained by the presence of the virulence-associated alleles for a subset of genes. Indeed, out of the major virulence loci identified by Saeij et al. [41], TgCkUg2 possessed the virulent allele for two loci (on chromosomes VIIa and XII) and the avirulent allele for two (on chromosomes VIIb and X). The gene responsible for the quantitative trait locus on VIIa is that encoding ROP18 [41,42], where genotype II is associated with mouse virulence, but no polymorphisms were seen for ROP18 in
TgCkUg2 - it was identical to Me49 and the Ugandan type II strains. SAG3 and ROP5 are the candidate genes on XII [41], where genotype III is the virulent type, and while no new SNPs were found for TgCkUg2 in the $\mathrm{SAG}_{3}$ gene, ROP 5 was one of the genes with the highest number of novel SNPs. The presence of six non-synonymous and three synonymous new SNPs suggests that $\mathrm{ROP}_{5}$ may be under selection in TgCkUg2. Additional genes identified through statistical dN/ $\mathrm{dS}$ analysis to be under selective pressure, or found to contain at least two novel coding SNPs in TgCkUg2, included those encoding GRA3 and several ROPs and SRSs (Tables 3 and 4), which may be important for virulence and host-pathogen interactions. Interestingly, one of the genes (551.moo237) found to be under selective pressure by $\mathrm{dN} / \mathrm{dS}$ analysis is located within the proposed virulence locus on chromosome XII, next to the candidate gene $\mathrm{ROP}_{5}$. While it is currently not possible to determine the exact genes responsible for the intermediate phenotype of this recombinant strain, the high density of SNP data from the current study will provide the basis for closer mapping of genotype-phenotype associations and genes under selection for niche adaptation within African isolates.

\section{Conclusions}

T. gondii is an important pathogen with unusual population structure. Although highly zoonotic, it is genetically conserved; thus, considerable depth of study is required to understand genotype phenotype relationships. There are three major outstanding questions about the population genetics of this parasite. Firstly, does recombination occur and, if so, why do clonal genotypes dominate? Secondly, what is the extent of allelic variation among strains? Finally, does allelic variation reflect biogeographical segregation and divergence or are there loci under selection that might dictate the parasite phenotype?

Our study advances understanding of all three of these questions. We find that TgCkUg2, a natural type II/III recombinant strain, has arisen via chromosome sorting and there is no evidence for recent intrachromosomal recombination. Detailed mapping revealed 1,252 unique SNPs within TgCkUg2, which place the divergence of this African strain in relation to the annotated genomes of the North Americanderived reference type II and III isolates at around $10^{4}$ years ago. Direct sequencing confirms the presence of unique SNPs in additional African isolates, implying that these polymorphisms represent local allelic variants. Estimation of $\mathrm{dN} / \mathrm{dS}$ ratios across the genome of TgCkUg2 provides evidence of loci under selection. The intermediate phenotype of the recombinant strain provides a reference point for interpretation of the role of candidate virulence genes.

The substantial body of data generated from this study will inform future strategies for analysis of parasite population structure, recombination frequency and gene flow. The exist- 
ence of the recombinant and closely related parental strains further provide a platform for further genotype-phenotype studies.

\section{Materials and methods Parasite isolation and maintenance}

Eight $T$. gondii strains were isolated in mice from Ugandan chickens. These strains are designated as TgCkUg 1, 2, 3, 5, 6, 7,8 and 9 obtained from chickens 1, 2, 17, 68, 70, 79, 81 and 82, respectively [19]. Strains were retrieved from liquid nitrogen, passaged once in Swiss-Webster mice (at the United States Department of Agriculture (USDA)) and homogenized brain material was thereafter injected into three inbred NMRI mice (at the Swedish Institute for Infectious Disease Control (SMI)) for each isolate. The mice were kept 6 weeks and thereafter put to death humanely. Heart, brain, quadriceps muscle and lung tissue were dissected for quantification of parasite burden, and parasites from the brain were transferred to human foreskin fibroblast cell culture. The brains were homogenized in $1 \mathrm{ml}$ sterile phosphate-buffered saline and subject to digestion in $0.5 \%$ trypsin-EDTA for 30 minutes at $37^{\circ} \mathrm{C}$, in order to release bradyzoites from their tissue cysts [46]. The parasites were kept in cell culture under standard conditions in Dulbecco's minimum essential medium with 10\% foetal bovine serum, 1\% HEPES and 1\% penicillin and streptomycin. Parasites were regularly passaged to new human foreskin fibroblast cells. All animal work was performed according to national and international guidelines.

\section{Whole genome sequencing of a natural recombinant strain}

Parasites of the TgCkUg2 strain were harvested from cell culture and intracellular parasites were released by needle passage and separated from host cells through centrifugation. DNA was extracted using the QIAamp DNA extraction kit (QIAgen $\mathrm{GmbH}$, Hilden, Germany) and adjusted to a concentration of $100 \mathrm{ng} / \mu \mathrm{l}$ to a total of $5 \mu \mathrm{g}$.

We generated 1,480,197 reads in total in three full runs of the 454GSFLX sequencer (Roche, Basel, Switzerland), which resulted in $328,210,041$ bases of sequence. In mapping to the Me49 sequence from ToxoDB v4.3, 49.7\% of the reads mapped to the genome using the default parameters of the mapping algorithm in the Newbler Software package (Roche); $7.6 \%$ of the reads did not map due to being in repetitive regions. The remaining $43 \%$ of the sequence contained either host sequence (54.7\%) or T. gondii sequence that fell below the mapping criteria (27.7\%). The remaining sequences are either missing from the reference or due to other contamination within the sample. After mapping we generated 64,721 contigs of $49,866,158 \mathrm{bp}$ in total. This is somewhat shorter than the reference $(60,179,518 \mathrm{bp})$ due to repetitive regions, which cannot be mapped to. The average coverage of the mapped portion of the genome was therefore $4 \times$.

\section{SNP analysis}

The sequence of TgCkUg2 was aligned to the annotated sequence of Me49 (ToxoDB v4.3), and thereafter to VEG, which had first been mapped to the Me49 sequence. SNPs were called when at least three reads, of which at least one was in each direction, called a SNP with $100 \%$ concordance. SNPs called in regions that mapped to several places in the genome or SNPs where all reads were not in agreement were dismissed. These strict criteria were used to prevent false SNP calling, but are likely to miss a large proportion of real polymorphisms. To assess this rate, each chromosome was queried for polymorphisms between VEG and Me49 in ToxoDB and compared with the SNPs detected in our analysis, and we found that the SNP detection rate was between 22 and $24 \%$.

The sequence data were analyzed in the sequence viewer and annotation tool Artemis after pre-processing with bespoke Perl scripts, and the annotation for Me49 (ToxoDB v4.3) was used. The three main SNP types are shown in green for $($ TgCkUg2 $=$ Me49 $) \neq$ VEG, blue for $($ TgCkUg2 $=$ VEG $) \neq$ Me49 and orange for TgCkUg2 $\neq(\mathrm{Me} 49=\mathrm{VEG})$. The SNPs were imported from Artemis to Excel for calculations and creation of the SNP density graphs (Additional data file 1). Since TgCkUg2 was a type II/III recombinant, it was not aligned on the whole genome level with the type I reference strain GT1 as this would have greatly increased the complexity of the analysis while adding little useful information. However, in the case of additional targeted sequencing of all Ugandan isolates, comparisons were made with all type I, II and III reference strains. The distribution of SNPs in TgCkUg was also mapped against maps of SNP dominance generated from reference strains as described below.

To compare the polymorphisms in TgCkUg2 to those in the three reference strains (type I, GT-1; type II, ME49; and type III, VEG), all predicted polymorphisms among the type I, II and III strains (kindly provided by Amit Bahl and David Roos, University of Pennsylvania) were used to create SNP type plots as follows. Starting from the left end of each chromosome, a running sum was computed at each SNP position; a type I SNP counted o, a type II SNP counted +1 , and a type III SNP counted -1 . This running sum was then plotted against the SNP position on the chromosome (see gray line in Figure 2 and Additional data file 2), and then overlaid to scale on the chromosome maps depicting the degree of similarity between SNPs in TgCkUg2 and type II and type III sequences. On these graphs, therefore, a straight line indicates a chromosomal region where type I SNPs predominate in the canonical type I, II and III strains, a rising line indicates an area of type II dominance, and a falling line indicates an area of type III dominance.

Detection of genes under selection was achieved through $\mathrm{dN} /$ $\mathrm{dS}$ analysis of all chromosomes using the maximum likelihood method as detailed by Yang [25] and implemented by the codeml program within the Paml (version $4 \mathrm{~b}$ ) software 
package. Pairwise comparisons of the three strains Me49, VEG and TgCkUg2 were undertaken with each comparison consisting of: a maximum likelihood pairwise comparison to estimate $\mathrm{dN} / \mathrm{dS}$ (omega); an equivalent maximum likelihood comparison, but with omega fixed at 1 ; and a likelihood ratio test to assess whether the estimated omega was significantly greater than 1, and thus indicative of positive selection. More detail and a full list of the genes under selection between any of the strains, including the Me49-VEG comparison, is available in Additional data file 3 .

\section{Divergence time estimate}

The type II and type III chromosomes of TgCkUg2 were used in parallel to calculate the time of the MRCA of UgII and Me49, and UgIII and VEG, respectively, using two different methodologies. For calculations based on the intron mutation rate for T. gondii, the quote of the intron minor SNP count divided by the intron length was divided by the mutation rate $1.94 \times 10^{-8}$ [26] for all chromosomes separately, and for the entire type II and III regions together. Intronic SNPs were defined as SNPs present in the mRNA, but not in the coding sequence, and the intron lengths were calculated analogously (data were retrieved from Artemis and exported to Excel). The minor SNP type was defined as SNPs against the background (that is, against Me49 on type II chromosomes and against VEG on type III chromosomes), and included novel SNPs.

For calculations based on the type II/III divergence time estimate of 150,000 years [27], the relationship between major and minor SNPs was assumed to be directly proportional to the relationship in time between the ancient and more recent splits. The recombination events between the ancestral strains that gave rise to the present lineages occurred much more recently then did the MRCA of all the present strains. Types II and III have therefore had much less time to diverge in the regions they inherited jointly in this cross - that is, where type I SNPs are predominant [13]. Therefore, this method is not applicable to regions without SNPs between types II and III, and this is why chromosomes Ia and XI and several regions of other chromosomes were excluded. Major SNPs were defined as SNPs against the non-background type (but excluding unique SNPs), and minor SNPs as in the above paragraph.

The $T$. gondii mutation rate on the genome level (including introns, exons and intergenic regions) was calculated, based on the time estimate results from the calculations based on the major-minor SNP relationship, by dividing the quote of the minor SNP count over the sequence length with the new time estimates. Raw data and calculations are provided in Additional data file 4.

\section{Targeted sequencing}

Sanger sequencing confirmed SNPs discovered by the 454 sequencing of $\mathrm{TgCkUg} 2$, and was used to investigate the genetic relationship of the Ugandan isolates and reference strains. PCR products were purified using a PCR purification kit (QIAgen) and sequenced using Big Dye Terminator on an ABIPrism sequencer. Reference sequences for GT1, Me49 and VEG were retrieved from ToxoDB. TgCkUg2, 5, 6 and 8 were sequenced for all loci, and the four remaining Ugandan type II strains were sequenced for selected loci where informative polymorphisms were found. Thirty-four different loci were investigated and the primers are listed in Additional data file 5 .

\section{Data deposition}

All contigs assembled from the 454 sequencing of TgCkUg2 have been submitted to the Toxoplasma database [14], together with information on all identified SNPs between TgCkUg2 and Me49 or VEG. Incorporation of these data is in progress. The sequence data generated through conventional sequencing from the eight Ugandan strains are available in GenBank, accession numbers [GenBank:FI274744] to [GenBank:FI274973].

\section{Phenotype in vivo and in vitro}

The parasite density in different mouse organs was determined in mice used for isolation purposes. Each isolate was retrieved from storage at the USDA and inoculated into one mouse, from which half the brain was shipped to the SMI. For each isolate, half a brain was homogenized in phosphate-buffered saline and inoculated into three mice, from which brain, heart, lung and quadriceps muscle were dissected, weighed and used for DNA-extraction. Q-PCR with primers 5'-GCTCCTCCAGCCGTCTTG-3' (forward), 5'-TCCTCACCCTCGCCTTCAT-3' (reverse), together with a 6-FAM labeled probe $5^{\prime}-$ AGGAGAGATATCAGGACTGTA-3' (designed by Daniel Palm, SMI) to target the approximately 300-fold repetitive region $\mathrm{AF} 146527$ [47]. $\mathrm{ROX}$ was used as a passive reference and the program used was 2 minutes at $50^{\circ} \mathrm{C}, 10$ minutes at $95^{\circ} \mathrm{C}$ followed by 45 cycles of $15 \mathrm{~s}$ at $95^{\circ} \mathrm{C}$ and 1 minute at $60^{\circ} \mathrm{C}$. All samples were analyzed in duplicate and, in addition, two negative controls and a triplicate tenfold dilution series used to generate the standard curve, ranging from 0.1 to 1,00o parasites/ $\mu$ l of DNA template, were included on every plate.

In vitro growth data were collected during the isolates' eighth passage in cell culture. The well-characterized laboratory strain Me49 was included as a reference and studied in parallel with the new isolates. All parasites were grown in T25 flasks in exactly $6 \mathrm{ml}$ of medium and, for each time point, $5 \mathrm{ml}$ were taken for DNA extraction and replaced with fresh medium; thus, only extracellular parasites were quantified. Growth was also continuously monitored in all passages by visual inspection and scoring of intracellular growth. 


\section{Abbreviations}

$\mathrm{dN} / \mathrm{dS}$ : non-synonymous to synonymous mutation rate; GRA: dense granule protein; MRCA: most recent common ancestor; Q-PCR: quantitative PCR; ROP: rhoptry protein; SMI: Swedish Institute for Infectious Disease Control; SNP: single nucleotide polymorphism; SRS: SAG 1 like surface antigen; USDA: United States Department of Agriculture.

\section{Authors' contributions}

ILB carried all studies on isolation, phenotyping and sequencing of Toxoplasma strains; advice and support was provided by JPD for Toxoplasma strain isolation and by JL for phenotyping studies. NH designed and implemented the 454 sequencing strategy and, together with KEA, assimilated genome data into contigs. NH, KEA and ILB undertook SNP mapping and analysis, ILB completed MRCA analysis, KEA completed the $\mathrm{dN} / \mathrm{dS}$ screen and JPB mapped data against SNP profiles from ToxoDB. JES and ILB conceived the study, and participated in its design and coordination. ILB and JES drafted the manuscript. All authors provided comments on the paper and read and approved the final manuscript.

\section{Additional data files}

The following additional data are available with the online version of this paper: graphs showing the distribution of SNPs called from the 454 whole genome sequencing of the recombinant Ugandan T. gondii strain TgCkUg2 (Additional data file 1); overlay pictures for all 14 chromosomes that compare the distribution of SNPs in TgCkUg2 to the dominant SNP types for the three Toxoplasma reference strains (Additional data file 2); a table showing maximum likelihood analysis of SNPs, listing loci where omega $(\mathrm{dN} / \mathrm{dS})$ is significantly greater than 1 (Additional data file 3); a table showing original data and calculations for divergence time, using either the intron mutation rate of $1.94 \times 10^{-8}$ or the MRCA estimate of 150,000 years for calibration of the molecular clock (Additional data file 4); a table of primers used for sequence-based genotyping of Toxoplasma isolates (Additional data file 5).

\section{Acknowledgements}

This work was funded by the AGAPE Marie Curie EST (MEST5043 I8) and by a small grant from NERC (MGF240). The authors would like to thank Dr Natrajan Sundar at USDA for preparation of infected mouse brains, $\mathrm{Dr}$ Chunlei Su at the University of Tennessee for sharing unpublished information on isolate genotypes, Margaret Hughes for carrying out the genome sequencing, and the ToxoDB team for their work with incorporation of the $\mathrm{TgCkUg2} \mathrm{data.}$

\section{References}

I. Smith JE: Tracking transmission of the zoonosis Toxoplasma gondii. Adv Parasitol 2009, 68:139-159.

2. Grigg ME, Suzuki Y: Sexual recombination and clonal evolution of virulence in Toxoplasma. Microbes Infect 2003, 5:685-690.

3. Khan A, Taylor S, Su C, Mackey AJ, Boyle J, Cole R, Glover D, Tang K, Paulsen IT, Berriman M, Boothroyd JC, Pfeffercorn ER, Dubey JP, Ajioka JW, Roos DS, Wooton JC, Sibley LD: Composite genome map and recombination parameters derived from three archetypal lineages of Toxoplasma gondii. Nucleic Acids Res 2005, 33:2980-2992.

4. Pfefferkorn ER, Kasper LH: Toxoplasma gondii: genetic crosses reveal phenotypic suppression of hydroxyurea resistance by fluorodeoxyuridine resistance. Exp Parasitol 1983, 55:207-218.

5. Pfefferkorn LC, Pfefferkorn ER: Toxoplasma gondii: genetic recombination between drug resistant mutants. Exp Parasitol 1980, 50:305-316.

6. Sibley LD, LeBlanc AJ, Pfefferkorn ER, Boothroyd JC: Generation of a restriction fragment length polymorphism linkage map for Toxoplasma gondii. Genetics 1992, 132:1003-1015.

7. Grigg ME, Bonnefoy S, Hehl AB, Suzuki Y, Boothroyd JC: Success and virulence in Toxoplasma as the result of sexual recombination between two distinct ancestries. Science 200I, 294: $161-165$

8. Khan A, Fux B, Su C, Dubey JP, Darde ML, Ajioka JW, Rosenthal BM, Sibley LD: Recent transcontinental sweep of Toxoplasma gondii driven by a single monomorphic chromosome. Proc Natl Acad Sci USA 2007, I 04: | 4872- | 4877.

9. Pena HF, Gennari SM, Dubey JP, Su C: Population structure and mouse-virulence of Toxoplasma gondii in Brazil. Int J Parasitol 2007, 38:56I-569.

10. Ajzenberg D, Banuls AL, Su C, Dumetre A, Demar M, Carme B, Darde ML: Genetic diversity, clonality and sexuality in Toxoplasma gondii. Int J Parasitol 2004, 34: I |85-। 196.

II. Dubey JP, Cortes-Vecino JA, Vargas-Duarte JJ, Sundar N, Velmurugan GV, Bandini LM, Polo LJ, Zambrano L, Mora LE, Kwok OC, Smith T, Su C: Prevalence of Toxoplasma gondii in dogs from Colombia, South America and genetic characterization of T. gondii isolates. Vet Parasitol 2007, I 45:45-50.

12. Lehmann T, Marcet PL, Graham DH, Dahl ER, Dubey JP: Globalization and the population structure of Toxoplasma gondii. Proc Natl Acad Sci USA 2006, 103: I |423- I | 428.

13. Boyle JP, Rajasekar B, Saeij JP, Ajioka JW, Berriman M, Paulsen I, Roos DS, Sibley LD, White MW, Boothroyd JC: Just one cross appears capable of dramatically altering the population biology of a eukaryotic pathogen like Toxoplasma gondii. Proc Natl Acad Sci USA 2006, 103:10514-10519.

14. ToxoDB [http://toxodb.org/toxo/]

15. Gajria B, Bahl A, Brestelli J, Dommer J, Fischer S, Gao X, Heiges M, lodice J, Kissinger JC, Mackey AJ, Pinney DF, Roos DS, Stoeckert C], Wang H, Brunk BP: ToxoDB: an integrated Toxoplasma gondii database resource. Nucleic Acids Res 2008:D553-556.

16. Khan A, Bohme U, Kelly KA, Adlem E, Brooks K, Simmonds M, Mungall K, Quail MA, Arrowsmith C, Chillingworth T, Churcher C, Harris D, Collins M, Fosker N, Fraser A, Hance Z, Jagels K, Moule S, Murphy L, O'Neil S, Rajandream MA, Saunders D, Seeger K, Whitehead S, Mayr T, Xuan X, Watanabe J, Suzuki Y, Wakaguri H, Sugano S, et al.: Common inheritance of chromosome la associated with clonal expansion of Toxoplasma gondii. Genome Res 2006, I6: I I 19-I I 25.

17. Sibley LD, Ajioka JW: Population Structure of Toxoplasma gondii: clonal expansion driven by infrequent recombination and selective sweeps. Annu Rev Microbiol 2008, 62:329-35I.

18. Velmurugan GV, Dubey JP, Su C: Genotyping studies of Toxoplasma gondii isolates from Africa revealed that the archetypal clonal lineages predominate as in North America and Europe. Vet Parasitol 2008, I 55:3 |4-318.

19. Lindstrom I, Sundar N, Lindh J, Kironde F, Kabasa JD, Kwok OC, Dubey JP, Smith JE: Isolation and genotyping of Toxoplasma gondii from Ugandan chickens reveals frequent multiple infections. Parasitology 2008, I35:39-45.

20. Swaminathan K, Varala K, Hudson ME: Global repeat discovery and estimation of genomic copy number in a large, complex genome using a high-throughput 454 sequence survey. $B M C$ Genomics 2007, 8:132.

21. Fichera ME, Roos DS: A plastid organelle as a drug target in apicomplexan parasites. Nature 1997, 390:407-409.

22. Kohler S, Delwiche CF, Denny PW, Tilney LG, Webster P, Wilson RJ, Palmer JD, Roos DS: A plastid of probable green algal origin in Apicomplexan parasites. Science 1997, 275: |485-I489.

23. Matsuzaki M, Kikuchi T, Kita K, Kojima S, Kuroiwa T: Large amounts of apicoplast nucleoid DNA and its segregation in Toxoplasma gondii. Protoplasma 2001, 2 18: |80-I91.

24. Ferguson DJ, Henriquez FL, Kirisits MJ, Muench SP, Prigge ST, Rice DW, Roberts CW, McLeod RL: Maternal inheritance and stagespecific variation of the apicoplast in Toxoplasma gondii dur- 
ing development in the intermediate and definitive host. Eukaryot Cell 2005, 4:8I4-826.

25. Yang Z: Computational Molecular Evolution Oxford: Oxford University Press; 2006.

26. Su C, Evans D, Cole RH, Kissinger JC, Ajioka JW, Sibley LD: Recent expansion of Toxoplasma through enhanced oral transmission. Science 2003, 299:4|4-4I6.

27. Morrison DA: How old are the extant lineages of Toxoplasma gondii? Parassitologia 2005, 47:205-2/4.

28. Toxoplasma Genome Map [http://toxomap.wustl.edu/]

29. Innes EA: Toxoplasmosis: comparative species susceptibility and host immune response. Comp Immunol Microbiol Infect Dis 1997, 20:131-138.

30. Frenkel JK: Pathophysiology of toxoplasmosis. Parasitol Today 1988, 4:273-278

31. Araujo F, Slifer T, Kim S: Chronic infection with Toxoplasma gondii does not prevent acute disease or colonization of the brain with tissue cysts following reinfection with different strains of the parasite. J Parasitol 1997, 83:521-522.

32. Dao A, Fortier B, Soete M, Plenat F, Dubremetz JF: Successful reinfection of chronically infected mice by a different Toxoplasma gondii genotype. Int J Parasitol 200 I, 31:63-65.

33. Dzitko K, Staczek P, Gatkowska J, Dlugonska H: Toxoplasma gondii: Serological recognition of reinfection. Exp Parasitol 2006, I I 2:134-137.

34. Aspinall TV, Guy EC, Roberts KE, Joynson DH, Hyde JE, Sims PF: Molecular evidence for multiple Toxoplasma gondii infections in individual patients in England and Wales: public health implications. Int J Parasitol 2003, 33:97-I03.

35. Ferreira Ade M, Vitor RW, Gazzinelli RT, Melo MN: Genetic analysis of natural recombinant Brazilian Toxoplasma gondii strains by multilocus PCR-RFLP. Infect Genet Evol 2006, 6:22-31.

36. Dubey JP, Sundar N, Pineda N, Kyvsgaard NC, Luna LA, Rimbaud E, Oliveira JB, Kwok OC, Qi Y, Su C: Biologic and genetic characteristics of Toxoplasma gondii isolates in free-range chickens from Nicaragua, Central America. Vet Parasitol 2006, I 42:47-53.

37. Beck HP, Blake D, Darde ML, Felger I, Pedraza-Diaz S, Regidor-Cerrillo J, Gomez-Bautista M, Ortega-Mora LM, Putignani L, Shiels B, Tait A, Weir W: Molecular approaches to diversity of populations of apicomplexan parasites. Int J Parasitol 2009, 39: $175-189$

38. Su C, Howe DK, Dubey JP, Ajioka JW, Sibley LD: Identification of quantitative trait loci controlling acute virulence in Toxoplasma gondii. Proc Natl Acad Sci USA 2002, 99:10753-10758.

39. Bromham L, Penny D: The modern molecular clock. Nat Rev Genet 2003, 4:216-224.

40. Zuckerkandl E, Pauling L: Molecules as documents of evolutionary history. I Theor Biol 1965, 8:357-366.

4I. Saeij JP, Boyle JP, Coller S, Taylor S, Sibley LD, Brooke-Powell ET, Ajioka JW, Boothroyd JC: Polymorphic secreted kinases are key virulence factors in toxoplasmosis. Science 2006, 314: 1780-1783.

42. Taylor S, Barragan A, Su C, Fux B, Fentress SJ, Tang K, Beatty WL Hajj HE, Jerome M, Behnke MS, White M, Wootton JC, Sibley LD: A secreted serine-threonine kinase determines virulence in the eukaryotic pathogen Toxoplasma gondii. Science 2006 314:1776-1780

43. Bradley PJ, Sibley LD: Rhoptries: an arsenal of secreted virulence factors. Curr Opin Microbiol 2007, 10:582-587.

44. Craver MP, Knoll LJ: Increased efficiency of homologous recombination in Toxoplasma gondii dense granule protein 3 demonstrates that GRA3 is not necessary in cell culture but does contribute to virulence. Mol Biochem Parasitol 2007, I 53: 149-157.

45. Jung C, Lee CY, Grigg ME: The SRS superfamily of Toxoplasme surface proteins. Int J Parasitol 2004, 34:285-296.

46. Dubey JP: Re-examination of resistance of Toxoplasma gondii tachyzoites and bradyzoites to pepsin and trypsin digestion. Parasitology 1998, I 1 6:43-50.

47. Homan WL, Vercammen M, De Braekeleer J, Verschueren H: Identification of a 200- to 300 -fold repetitive 529 bp DNA fragment in Toxoplasma gondii, and its use for diagnostic and quantitative PCR. Int J Parasitol 2000, 30:69-75. 